\begin{tabular}{|c|c|}
\hline Title & A reduced model for finite element analysis of steel laminations \\
\hline Author(s) & Igarashi, Hajime; W atanabe, Kota; Kost, A rnulf \\
\hline Citation & $\begin{array}{l}\text { IEEE transactions on magnetics, 42(4), 739-742 } \\
\text { https:/doi. org/10.1109// MA G.2006.872470 }\end{array}$ \\
\hline Issue Date & $2006-04$ \\
\hline Doc URL & http://hdl.handle.net/2115/8517 \\
\hline Rights & $\begin{array}{l}\text { (c) } 2006 \text { IEEE. Personal use of this material is permitted. However, permission to reprint/republish this material for } \\
\text { advertising or promotional purposes or for creating new collective works for resale or redistribution to servers or lists, } \\
\text { or to reuse any copyrighted component of this work in other works must be obtained from the IEEE }\end{array}$ \\
\hline Type & article \\
\hline File Information & 01608312.pdf \\
\hline
\end{tabular}

Instructions for use 


\title{
A Reduced Model for Finite Element Analysis of Steel Laminations
}

\author{
Hajime Igarashi ${ }^{1}$, Kota Watanabe ${ }^{1}$, and Arnulf Kost ${ }^{2}$ \\ ${ }^{1}$ Graduate School of Information Science and Technology, Hokkaido University, Sapporo 060-0814, Japan \\ ${ }^{2}$ Brandenburg University of Technology Cottbus, Germany
}

\begin{abstract}
This paper describes a method which reduces the number of unknowns in the finite element analysis of steel laminations. In the present method, the global variation in the electromagnetic field is assumed to be piecewise linear or polynomial while periodical, local variation is analyzed by the finite element method. This method allows us to make accurate analysis of the field including edge effects in steel laminations with low memory consumption and short computing time.
\end{abstract}

Index Terms-Eddy currents, finite element method, lamination stack.

\section{INTRODUCTION}

$\mathbf{I}$ $\mathrm{N}$ the steel lamination with insulated spacing, eddy currents cannot form global circulation in the direction perpendicular to their faces. The eddy current loss can, therefore, be effectively reduced in comparison with the case of steel bulk. For this reason, the steel lamination is frequently used in the electromagnetic energy conversion systems such as transformers and motors.

It is important to make numerical analysis of electromagnetic fields in those energy conversion systems in the design processes. However, there are severe difficulties in the numerical analysis due to the lamination structure. For example, let us consider the situation in which high-frequency magnetic field is applied in parallel with the face of steel lamination. The eddy currents then circularly flow in each cross section of the layer. When analyzing this field using finite element method, the element size must be sufficiently smaller than the skin depth which could be much smaller than the thickness of the layer. Consequently, the number of unknowns would be beyond the limit of available memory size.

Homonization methods have been proposed to overcome this difficulty [1]-[3]. In this approach, the electromagnetic fields in the steel laminations are assumed to vary only in the direction of thickness. This leads to the one-dimensional Maxwell equation which can effectively be solved. This assumption gives, however, errors near the steel edges. Since the edge effects cannot be ignored in, for example, the teeth of a motor and MRI magnets, this drawback should be remedied.

This paper introduces a new method for effective analysis of steel lamination. In the present method, the global variation in the electromagnetic fields is assumed to be piecewise linear or polynomial whereas local periodical variation is analyzed by the three-dimensional finite element method without assumption of one-dimensionality.

\section{NUMERICAL METHOD}

Let us consider the steel lamination shown in Fig. 1, which would be a part of an electromagnetic system. To analyze the electromagnetic field in this steel lamination, we solve the

Digital Object Identifier 10.1109/TMAG.2006.872470

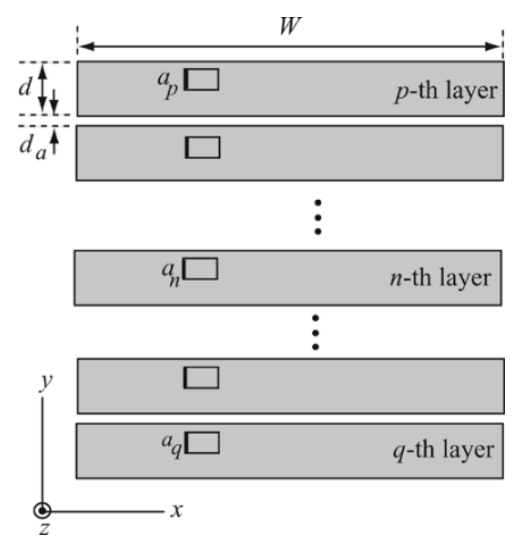

Fig. 1. Cross section of steel lamination. The dark rectangles represent steel layers. The gap between the steel layers is insulated. The small rectangles in the steel layers represent brick elements. The unknown vector potentials, $a, a_{p}$, and $a_{q}$, are assigned to the edges whose local positions in the layers are identical.

Maxwell equation under the quasistatic approximation using finite element method. We consider here a linear problem for simplicity although the present method could be applied to nonlinear problems. When using the A method, the system equation is given by

$$
\nabla \times(\nu \nabla \times \boldsymbol{A})+\mathrm{j} \omega \sigma \boldsymbol{A}=\boldsymbol{J}
$$

where $\nu$ and $\sigma$ are the magnetic reluctivity and electric conductivity, respectively. It is assumed that the steel lamination and insulation gaps are discretized using the brick edge-based elements. The discretized functional would be written in the following form:

$$
\mathcal{L}=\frac{1}{2} \sum_{i} \sum_{j} k_{i j} a_{i} a_{j}-\sum_{j} J_{j} a_{j}
$$

where $a_{j}$ denotes the projection of $\boldsymbol{A}$ to edge $j$. From the equations $\partial \mathcal{L} / \partial a_{i}=0$ for all edges $i$, we obtain the system of linear equations

$$
\left[k_{i j}\right]\left\{a_{i}\right\}=\left\{J_{i}\right\} .
$$

Next we consider the properties of the electromagnetic field in the steel lamination and insulation gaps. Due to the periodical structure of the steel lamination, the electromagnetic field would have a modulated periodical variation in $y$-direction. The 


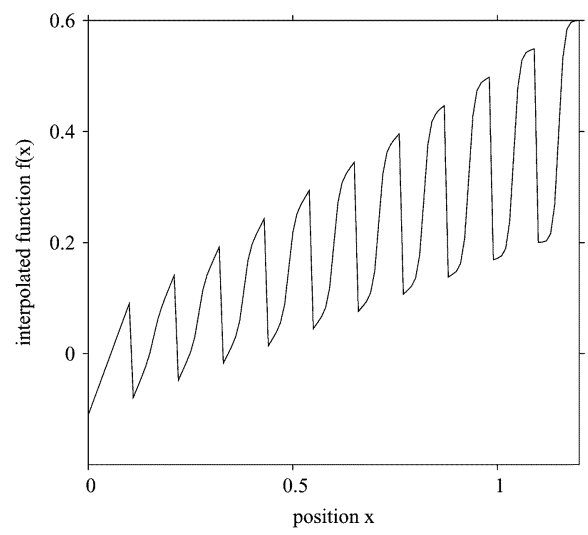

Fig. 2. An example of waveform expressed by the present method.

characteristic length of the modulation, caused by ambient materials and current sources, can be assumed to be sufficiently longer than the steel layer thickness $d$. We assume here that the long variation is piecewise linear between the $p$ th and $q$ th layers. Moreover, we consider the unknowns $a$ whose local position in the $p$ th and $q$ th layers as well as an intermediate $n$th layer are identical. Then the unknowns in these layers can be written in the form

$$
a_{n}=\xi a_{p}+(1-\xi) a_{q}
$$

where $\xi=(n-q) /(p-q)$. The unknowns $a$ in the insulation gap can be expressed in the similar form. For a continuous system, the above approximation for the long variation with periodical modulation would be written as

$$
\boldsymbol{A}(x, y+n \delta, z)=\xi \boldsymbol{A}(x, y+p \delta, z)+(1-\xi) \boldsymbol{A}(x, y+q \delta, z)
$$

where $0 \leq y \leq d, \delta=d+d_{a}$.

An example of waveform expressed by this approximation is shown in Fig. 2, where the first (left) and last (right) periods correspond to the $p$ th and $q$ th layers, and the intermediate periods are interpolated by the present method. The air gaps between the steel layers are neglected here, thus the resultant function $f(x)$ is continuous everywhere. The magnetic fields are also expressed in the similar form.

Using (4), $a$ in the intermediate steel layers and insulation gaps can be eliminated. The reduced system of linear equations can be obtained by substituting (4) into the discretized functional (2), and then differentiating it with respect to the remained unknowns. Consequently, the stiffness matrix is modified as follows:

if the edges $e$ and $e^{\prime}$ have their corresponding edges $(p, q),\left(p^{\prime}, q^{\prime}\right)$ in the $p$ th and $q$ th layers, then

$$
\begin{aligned}
& k_{p p^{\prime}} \leftarrow k_{p p^{\prime}}+\xi_{e} \xi_{e^{\prime}} k_{e e^{\prime}}, \\
& k_{p q^{\prime}} \leftarrow k_{p q^{\prime}}+\xi_{e}\left(1-\xi_{e^{\prime}}\right) k_{e e^{\prime}}, \\
& k_{p^{\prime} q} \leftarrow k_{p^{\prime} q}+\left(1-\xi_{e}\right) \xi_{e^{\prime}} k_{e e^{\prime}}, \\
& k_{q q^{\prime}} \leftarrow k_{q q^{\prime}}+\left(1-\xi_{e}\right)\left(1-\xi_{e^{\prime}}\right) k_{e e^{\prime}}
\end{aligned}
$$

and $k_{p^{\prime} p}=k_{p p^{\prime}}, k_{q^{\prime} p}=k_{p q^{\prime}}, k_{q p^{\prime}}=k_{p^{\prime} q}, k_{q^{\prime} q}=k_{q q^{\prime}}$, if the edge $e$ has its corresponding edges $(p, q)$, but it is not the case for $e^{\prime}$, then

$$
k_{p e^{\prime}} \leftarrow k_{p e^{\prime}}+\xi_{e} k_{e e^{\prime}}, \quad k_{q e^{\prime}} \leftarrow k_{q e^{\prime}}+\left(1-\xi_{e}\right) k_{e e^{\prime}}
$$

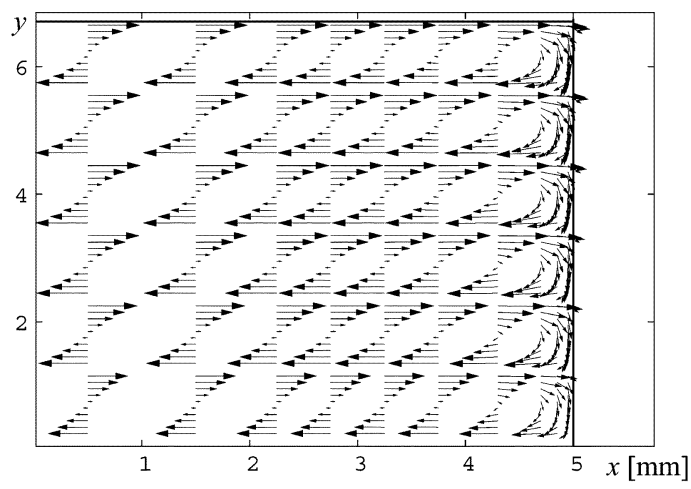

Fig. 3. Eddy current in the right half of steel lamination. $m=6$.

and $k_{e^{\prime} p}=k_{p e^{\prime}}, k_{e^{\prime} q}=k_{q e^{\prime}}$. Moreover, the source term is modified as

$$
J_{p} \leftarrow J_{p}+\xi_{e} J_{e}, \quad J_{q} \leftarrow J_{q}+\left(1-\xi_{e}\right) J_{e}
$$

if the edge $e$ has its corresponding edges $(p, q)$. Note here that we do not need create the global matrix for the original model, because these procedures can be performed for each element matrix.

The above reduction can also be realized using polynomial interpolation of higher order. However, it is recommended to use linear interpolation since the higher-order interpolation makes the global matrix denser. In practical situations, the steel lamination would be divided into some sub-domains for each of which this method is applied.

\section{NUMERICAL RESULTS}

In the following numerical tests, the linear interpolation (4) is employed for the present method. The domains are subdivided into brick elements and the number of elements in $y$-direction in each steel layer is set to 10 . The number of steel layers is denoted by $m$. Unless notice is given, the physical parameters are as follows: $f=500[\mathrm{~Hz}], \sigma=10^{7}[\mathrm{~S} / \mathrm{m}], \mu=1000 \mu_{0}$ for steel sheets, $d=1[\mathrm{~mm}], d_{a}=0.1[\mathrm{~mm}]$.

\section{A. Rectangular Cylinder}

Time-harmonic, uniform magnetic field perpendicular to the cross section of the lamination (i.e., in $z$-direction in Fig. 1) is applied to the steel lamination with $m$ layers surrounded by air region. The conductivity $\sigma$ which is uniform in the steel layer are linearly increased from $5 \times 10^{7}$ at the bottom layer to $1 \times 10^{7}$ $[\mathrm{S} / \mathrm{m}]$ at the top layer, as in [1]. The layers $p$ and $q$ correspond to the bottom and top layers, respectively. The system is assumed to be infinitely long in $z$-direction. Three dimensional finite element method is used for the analysis of the electromagnetic field in a slice of the system. Due to symmetry in the $x$-direction, only the upper right portion is analyzed. The number of elements in each direction is $n_{x}=10, n_{z}=1$, and $m=6$.

The eddy current distribution obtained by the present method is shown in Fig. 3. It was found that this distribution is very similar to the one obtained for the original model. In fact, there was no errors in the eddy current loss. The magnetic flux densities are plotted along the $y$-axis in Fig. 4. Although there are some discrepancies locally, overall agreement seems satisfactory.

Next we consider the computational cost and memory usage of the present method. The problem here is similar to the above 


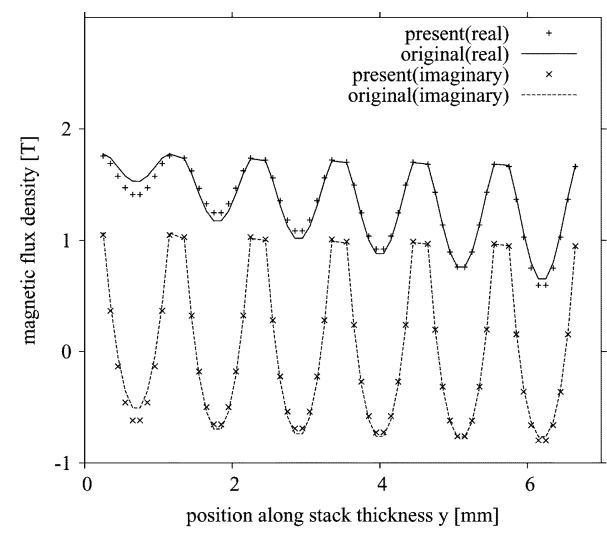

Fig. 4. Magnetic induction $B_{z}$ in steel lamination. $m=6, W=5[\mathrm{~mm}]$.

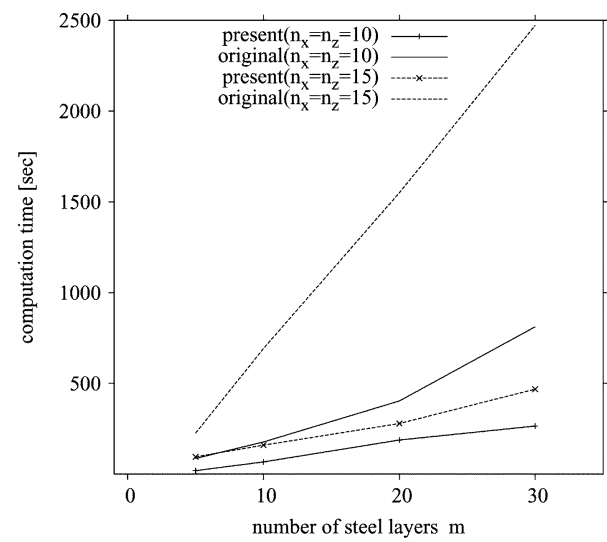

(a)

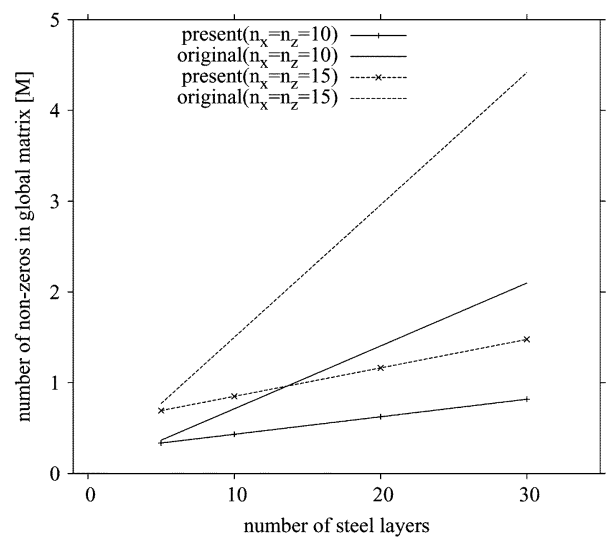

(b)

Fig. 5. Comparison of computation time and memory usage. (a) Computation time. (b) Memory usage.

problem but with uniform conductivity. The number of elements is $n_{x}=n_{z}$ for $x$ and $z$-directions, whereas $m=10$. The computation time and memory usages are shown in Fig. 5. It can be seen that the present method can effectively reduce memory usage when the number of steel layers increases. Moreover, as the number of elements in each steel layer increase, reduction of computation time and memory usage of the present method becomes remarkable.

It is found that the maximum band width of the original method keeps constant when the number of steel sheets $m$ increases while that of the present method grows. For example,

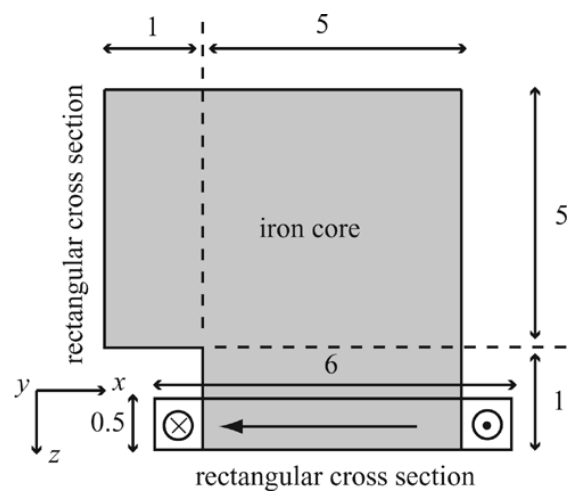

Fig. 6. Top view of $1 / 8$ model of iron core. unit [mm]

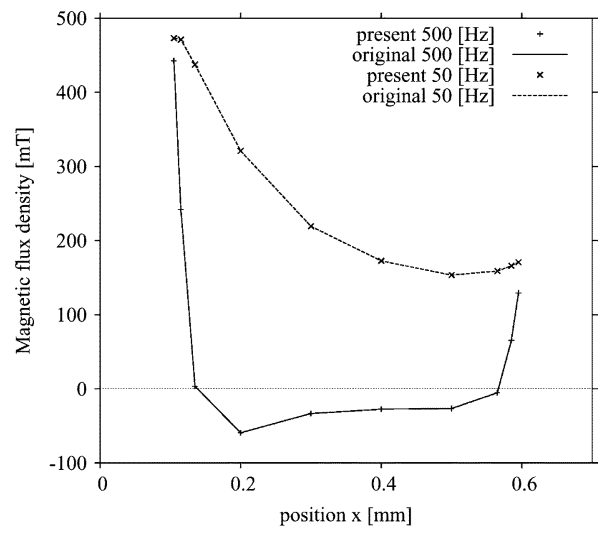

Fig. 7. Magnetic flux density $B_{z}$ in the cross section.

when $n_{x}=n_{z}=15$, the former is 29 whereas the latter increases from 150 to 410 , for $m=10$ and 30, respectively. In the present method, the unknowns in the $p$ th and $q$ th layers are connected each other in the system matrix. Moreover, the unknowns in the air region adjacent to the stack-edge surface are connected to ones in these two layer. This is the reason for the increase in the maximum band width. In the computer implementation, therefore, the memory allocation for nonzero terms should be adequately organized in order to consider the variation in the band width.

\section{B. Iron Core With Coil}

For the next numerical test, we consider the $1 / 8$ model of an iron core with a rectangular cross section, shown in Fig. 6, which can be regarded as a simple model of a transformer. The steel sheets are on the $x-z$ plane and other computation parameters are: $n_{x}=n_{z}=14, m=10$. The coil current modeled in terms of the current vector potential as $\boldsymbol{J}=\operatorname{rot} \boldsymbol{T}$.

Since all the steel sheets have the same eddy current distribution in this situation, we found almost no errors in eddy current distribution and total flux passing through the cross section of the iron core. Fig. 7 depicts the profiles of $B_{z}$, which proves the coincidence of the magnetic field obtained by the present and original model. When $f=500[\mathrm{~Hz}]$, skin depth, 0.23 [mm], is shorter than the thickness of the steel sheet. Thus the magnetic flux exists almost only in the edge layer. In contrast, it can penetrate the whole region of the cross section when $f=50[\mathrm{~Hz}]$ whose skin depth, $0.73[\mathrm{~mm}]$, is now comparable with the thickness of the steel sheet. 


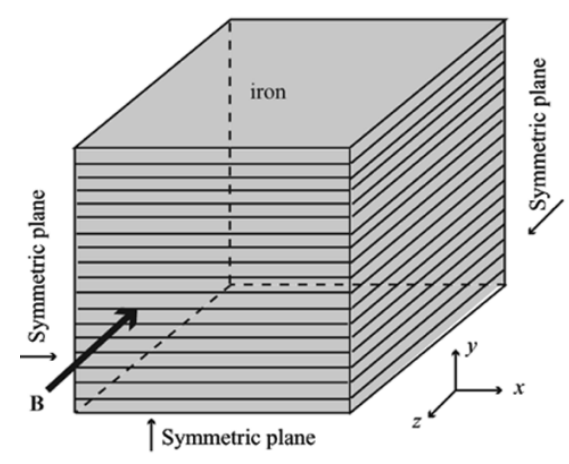

Fig. 8. 1/8 model of steel cube with external magnetic field.

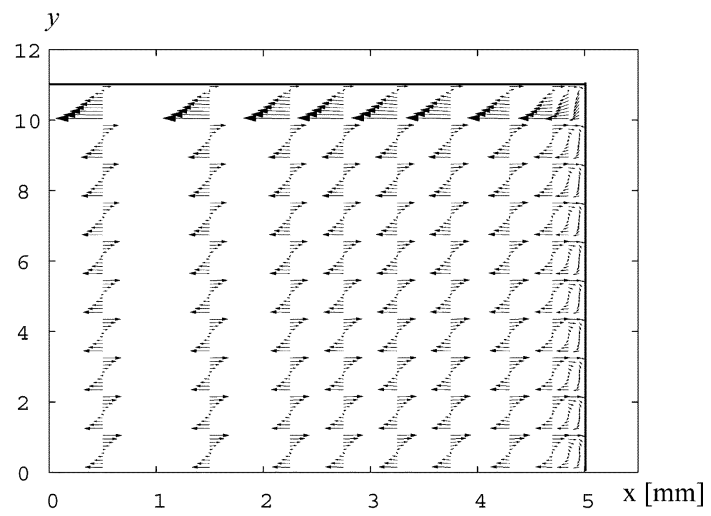

Fig. 9. Eddy current distribution.

\section{Steel Cube}

The last problem includes a steel cube composed of steel sheets immersed in an alternative magnetic field in $z$-direction, shown in Fig. 8. Due to geometrical symmetry, 1/8 of the whole region is modeled. In contrast to the previous problems, the electromagnetic field has three dimensional structure and has strong edge effect in the upper steel sheets. Since the present method would not be adequate for modeling of rapid changes in electromagnetic fields, the top steel sheet is modeled in a conventional way while the layers between the second top and bottom ones are modeled using the present method.

The eddy current distribution obtained by the present method is shown in Fig. 9, which was found to be in good agreement with that obtained using the original model. The strong eddy current flows in the top steel layer. Fig. 10 shows the profile of $B_{z}$ along the stack thickness. We can find the good coincidence between the values obtained using the present and original models.

\section{Discussions}

When using the present method, the finite elements in the air region adjacent to the stack-edge boundary must be conformable to the elements in the steel sheets. Although the latter elements can be eliminated using the present method, the former must be treated in a conventional way. The relatively large maximum band width mainly comes from this fact. When the air

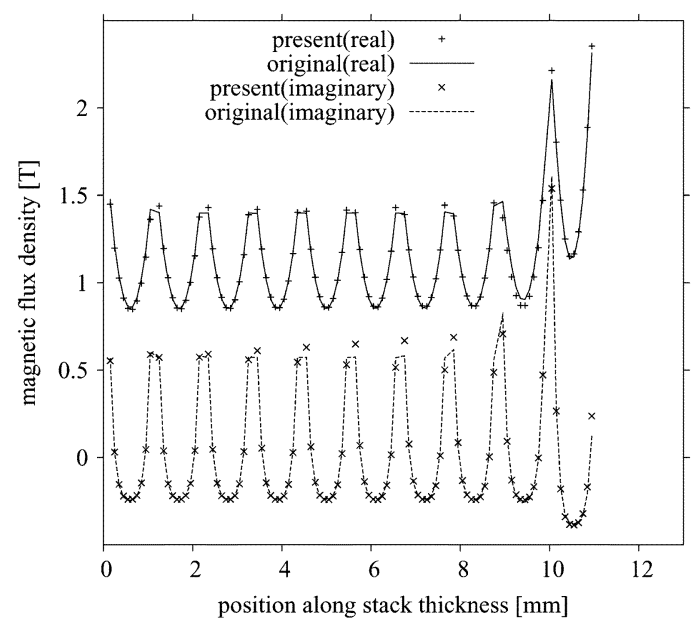

Fig. 10. Magnetic flux density $B_{z}$ in the cross section.

region surrounding the stack region is also modeled using the present method, this difficulty can be overcome. However, it is found out that this extended approximation is valid only when the field in each steel sheet is almost identical. It is a future work to improve this point. Moreover, applying the present method for more practical problems and comparison of the numerical results with experimental ones are also future works.

It is expected that no significant changes are necessary to apply the present method for problems with nonlinear and anisotropic materials. Moreover, the present method could be applied for the A-V method, which has better convergence than the A method [4]. These expectations should be validated in other works.

\section{CONCLUSION}

In this paper, a method to reduce the number of unknowns in the finite element analysis of steel laminations has been described. The present method allows us to reduce the memory consumption and computing time. In the rectangular cylinder and iron core models, the accuracy of the present method is satisfactory. When there is rapid changes in the electromagnetic fields in the end layers, they should be modeled in a conventional way, whereas the inner sheets can be modeled using the present method.

\section{REFERENCES}

[1] P. Dular, J. Gyselinck, C. Geuzaine, N. Sadowski, and J. P. A. Bastos, "A 3-D magnetic vector potential formulation taking eddy current in lamination stacks into account," IEEE Trans. Magn., vol. 39, no. 3, pp. 1424-1427, May 2003.

[2] L. Krähenbühl, P. Dular, T. Zeidan, and F. Buret, "Homogenization of lamination stacks in linear magnetodynamics," IEEE Trans. Magn., vol. 40, no. 2, pp. 912-915, Mar. 2004.

[3] K. Muramatsu, T. Okitsu, H. Fujitsu, and F. Shimanoe, "Method of nonlinear magnetic field analysis taking into account of eddy current in laminated core," IEEE Trans. Magn., vol. 40, no. 2, pp. 896-899, Mar. 2004.

[4] H. Igarashi and T. Honma, "Convergence of preconditioned conjugate gradient method applied to driven microwave problems," IEEE Trans. Magn., vol. 39, no. 3, pp. 1705-1708, May 2003.

Manuscript received June 24, 2005 (e-mail: igarashi@ssi.ist.hokudai.ac.jp). 